\title{
In silico toxicity prediction of 1-phenyl-1-(quinazolin-4-yl) ethanol compounds by using Toxtree, pkCSM and preADMET
}

\author{
Yeni", Supandi, Fajar Merdekawati \\ Department of Pharmaceutical Chemistry, Universitas Muhammadiyah Prof. DR. HAMKA \\ Jl. Delima II/IV, Jakarta 13460, Indonesia
}

Submitted: 05-04-2018

Reviewed: 05-06-2018

Accepted: 02-11-2018

\begin{abstract}
The 1-phenyl-1-(quinazolin-4-yl) ethanol compounds are alkaloids of quinozoline class found in many Hydrangeaceae families. A survey revealed that most of the identified quinazoline derivatives have anticancer activity. Toxicity prediction of 1-phenyl-1-(quinazolin-4-yl) ethanols compounds were performed to obtain the best three compounds with high activity and the lowest toxicity. Toxicity prediction was conducted using Toxtree, pkCSM and PreADMET. The 2D structure of compounds were formed using ChemDraw. The decision tree approach was used in Toxtree application with endpoints including Cramer rules, Kroes TTC, carcinogenicity (genotoxic and non genotoxic) and in vitro mutagenicity. Graph based signature was used in pkCSM application with endpoints including mutagenicity, maximum daily dose, $\mathrm{LD}_{50}$ and hepatotoxicity. In PreADMET application, a method based on drugs similarity and ADMET properties was used with endpoints including mutagenicity, carcinogenicity to rat and mice. The results of data analysis showed that the best three anticancer compounds that have high activity and the lowest toxicity are compounds 14, 16 and 19.
\end{abstract}

Keywords: toxicity prediction, in silico, 1-phenyl-1-(quinazolin-4-yl) ethanol compounds, Toxtree, pkCSM, PreADMET

\footnotetext{
*Corresponding author:

Yeni

Department of Pharmaceutical Chemistry

Universitas Muhammadiyah Prof. DR. HAMKA

Jl. Delima II/IV, Jakarta 13460, Indonesia

Email: yeni@uhamka.ac.id
} 


\section{INTRODUCTION}

Cancer is a chronic disease that causes death number two in the world. In 2012, cancer is responsible for the deaths of around 8.2 million people worldwide. The cases of cancer can increase up to 50\% in 2020 (Parameshwar et al., 2016). The high number of deaths caused by cancer led to studies on anticancer compounds expand.

The 1-phenyl-1-(quinazolin-4-yl) ethanol compounds are alkaloids of quinazolin group which are widely present in Hydrangeaceae family (Ajani et al., 2016). The most of quinazolin derivatives have been identified as having biological activities such as anticancer, antioxidant, antiviral, anticonvulsant, antiinflammatory, antituberculous, anti-HIV, analgesic, and antimicrobial (Faraj et al., 2014). Kuroiwa et al. (2015) have conducted a Quantitative Structure and Activity Relationships (QSAR) study and in vitro testing of 1-phenyl-1-(quinazolin-4-yl) ethanol compounds. The in vitro testing result of cell line A549 (lung) obtained a value of biological activity of $\mathrm{IC}_{50}$ which showed that 1-phenyl-1-(quinazolin-4-yl) ethanol compounds have potential as anticancer. The compounds have an anticancer mechanism through the binding of tubulin which binds to colchicine. It inhibits the binding of tubulin molecule and microtubule resulting polymerization in microtubules or failure of microtubule formation in cancer cells.

In silico toxicity prediction is a type of toxicity assessment using computational resources (algorithms, softwares and data) to organize, analyze, modeling, simulate, visualize, or predict chemical toxicity (Raies and Bajic, 2016). In silico toxicity prediction of 1-phenyl-1-(quinazolin-4yl) ethanol compounds was performed using Toxtree, pkCSM, and preADMET.

Toxtree is designed to estimate toxic hazards using decision tree approach. Decision tree uses the method based on Structural Alerts (SA) and QSAR. The method has a role to designate the potential of toxic chemicals (Benigni et al., 2008). The performance of Toxtree in the external validation dataset showed an accuracy of $70 \%$ and a sensitivity of $78.3 \%$ in the carcinogenicity test and an accuracy of $78 \%$ for the mutagenicity test (Valerio, 2009). Toxtree represents endpoints of different toxicities, i.e. Cramer rule, Kroes TTC, carcinogenicity (genotoxic and non genotoxic) and in vitro mutagenicity (Ames test).

PkCSM (Predicting Small-Molecule Pharmacokinetic and Toxicity Properties Using GraphBased Signatures) is a method for predicting and optimizing pharmacokinetic properties and toxicity properties. It use graph-based signatures approach. pkCSM adapted the cut off scanning concept to develop a predictive model of ADMET (Absorption, Distribution, Metabolism, Excretion, Toxicity) properties for drug development. The performance of pkCSM software in the external validation dataset showed an accuracy of $83.8 \%$ in the mutagenicity test. There are several endpoints of pkCSM i.e. $\mathrm{LD}_{50}$, ames test, maximum daily dose, and hepatotoxic (Pires et al., 2015).

PreADMET (Prediction of ADME/Tox) developed a fast and reliable method to predict the similarity of drugs and ADMET properties. This application can calculate more than 900 molecular descriptors including constitutional, topological, electrostatic, physico-chemical and geometric descriptor to predict ADME (Absorption, Distribution, Metabolism, Excretion) properties. PreADMET collects databases containing ADME and toxicities data to train physiologically-based pharmacokinetics model tissues and toxicity predictions. PreADMET provided $62.5 \%$ accuracy and $52.2 \%$ sensitivity for carcinogenicity test (Zhang et al., 2017). PreadMET predicts toxicity based on Ames mutagenity parameters. The actual value of the prediction is "positive" or "negative". The carcinogenicity is predicted based on the structure results which is constructed from NTP (National Toxicology Program) data and US FDA (US Food and Administration). It is the result of in vivo carcinogenicity test in rat for two years (Riju et al., 2010).

Many of bioactive compounds have been shown anticancer activity but their utilization is limited due to their side effects and high toxic effects, that are very dangerous and life-threatening effect (Priyanto, 2015). Therefore, in silico toxicity prediction of 1-phenyl-1-(quinazolin-4-yl) ethanol compounds was performed before in vitro and in vivo testing to minimize the number of test compounds and test animals in the following tests. 


\section{MATERIALS AND METHODS \\ Materials}

The softwares used in this research were ChemDraw 2016 (http: //scistore.cambridgesoft.com/) (License Code: 338-284099-4415), Openbabel GUI 2.4.1 (https://sourceforge.net), pkCSM ( http://biosig.unimelb.edu.au/pkcsm), Toxtree version 2.6.6 (http: //toxtree.sourceforge. net/) and preADMET (http://preadmet.bmdrs.kr). The materials used in this research were the $2 \mathrm{D}$ structures and $\mathrm{IC}_{50}$ value of 44 compounds of 1-phenyl-1-(quinazolin4-yl) ethanol which have been synthesized by Kuroiwa et al., (2015). IC $\mathrm{IC}_{50}$ value of 1-phenyl-1(quinazolin-4-yl) ethanol compounds are presented in Table I.

Table I. IC I0 $_{50}$ of 1-phenyl-1-(quinazolin-4-yl) ethanol compounds (Kuroiwa et al., 2015)

\begin{tabular}{|c|c|c|c|c|c|}
\hline $\begin{array}{l}\text { Compound } \\
\text { Number }\end{array}$ & $\begin{array}{l}\text { Name of The } \\
\text { Compounds }\end{array}$ & $\begin{array}{l}\mathrm{IC}_{50} \\
(\mu \mathrm{M})\end{array}$ & $\begin{array}{l}\text { Compound } \\
\text { Number }\end{array}$ & $\begin{array}{l}\text { Name of The } \\
\text { Compounds }\end{array}$ & $\begin{array}{l}\mathrm{IC}_{50} \\
(\mu \mathrm{M})\end{array}$ \\
\hline $1 \mathrm{a}$ & $\begin{array}{l}1-(4- \\
\text { methoxyphenyl)-1- } \\
\text { (quinazolin-4- } \\
\text { yl)ethan-1-ol }\end{array}$ & 0.27 & 9 & $\begin{array}{c}1-(2- \\
\text { chloroquinazolin-4- } \\
\text { yl)-1-(4- } \\
\text { methoxyphenyl)ethan } \\
\text {-1-ol }\end{array}$ & 2.0 \\
\hline $1 b$ & $\begin{array}{l}\text { 1-(4-fluorophenyl)-1- } \\
\text { (quinazolin-4- } \\
\text { yl)ethan-1-ol }\end{array}$ & $>25$ & 14 & $\begin{array}{c}1-(4- \\
\text { methoxyphenyl)-1- } \\
\text { (2-methylquinazolin- } \\
\text { 4-yl)ethan-1-ol }\end{array}$ & 0.053 \\
\hline $1 \mathrm{c}$ & $\begin{array}{l}\text { 1-(4-chlorophenyl)- } \\
\text { 1-(quinazolin-4- } \\
\text { yl)ethan-1-ol }\end{array}$ & $>25$ & 15 & $\begin{array}{c}1-(2- \\
\text { cyclohexylquinazolin } \\
-4-y 1)-1-(4- \\
\text { methoxyphenyl)etha } \\
\text { n-1-ol }\end{array}$ & 0.1 \\
\hline $1 d$ & $\begin{array}{l}\text { 1-(quinazolin-4-yl)- } \\
\text { 1-(4- } \\
\text { (trifluoromethyl)phen } \\
\text { yl)ethan-1-ol }\end{array}$ & $>25$ & 16 & $\begin{array}{c}1-(4- \\
\text { methoxyphenyl)-1- } \\
(2- \\
\text { (trichloromethyl)quin } \\
\text { azolin-4-yl)ethan-1- } \\
\text { ol }\end{array}$ & 0.038 \\
\hline 1f & $\begin{array}{l}\text { 1-(4-ethoxyphenyl)- } \\
\text { 1-(quinazolin-4- } \\
\text { yl)ethan-1-ol }\end{array}$ & 0.30 & 17 & $\begin{array}{c}1-(2- \\
\text { chloroquinazolin-4- } \\
\text { yl)-1-(4- } \\
\text { methoxyphenyl)etha } \\
\text { n-1-ol }\end{array}$ & 0.027 \\
\hline $1 \mathrm{~g}$ & $\begin{array}{l}\text { 1-(4-(tert- } \\
\text { butoxy)phenyl)-1- } \\
\text { (quinazolin-4- } \\
\text { yl)ethan-1-ol }\end{array}$ & $>25$ & 18 & $\begin{array}{l}\text { (R)-4-(1-hydroxy-1- } \\
\text { (4- } \\
\text { methoxyphenyl)ethyl } \\
\text { )quinazolin-2(1H)- } \\
\text { one }\end{array}$ & $>25$ \\
\hline $1 \mathrm{~h}$ & $\begin{array}{l}\text { 1-(quinazolin-4-yl)- } \\
\text { 1-(4- } \\
\text { (trifluoromethoxy)ph } \\
\text { enyl)ethan-1-ol }\end{array}$ & $>25$ & 19 & $\begin{array}{c}(\mathrm{R})-1-(4- \\
\text { methoxyphenyl)-1- } \\
(2- \\
\text { methoxyquinazolin- } \\
\text { 4-yl)ethanol }\end{array}$ & 0.058 \\
\hline $1 \mathrm{i}$ & $\begin{array}{c}1-(3- \\
\text { methoxyphenyl)-1- }\end{array}$ & $>25$ & 20 & $\begin{array}{c}\text { (R)-1-(2- } \\
\text { ethoxyquinazolin-4- }\end{array}$ & 0.34 \\
\hline
\end{tabular}




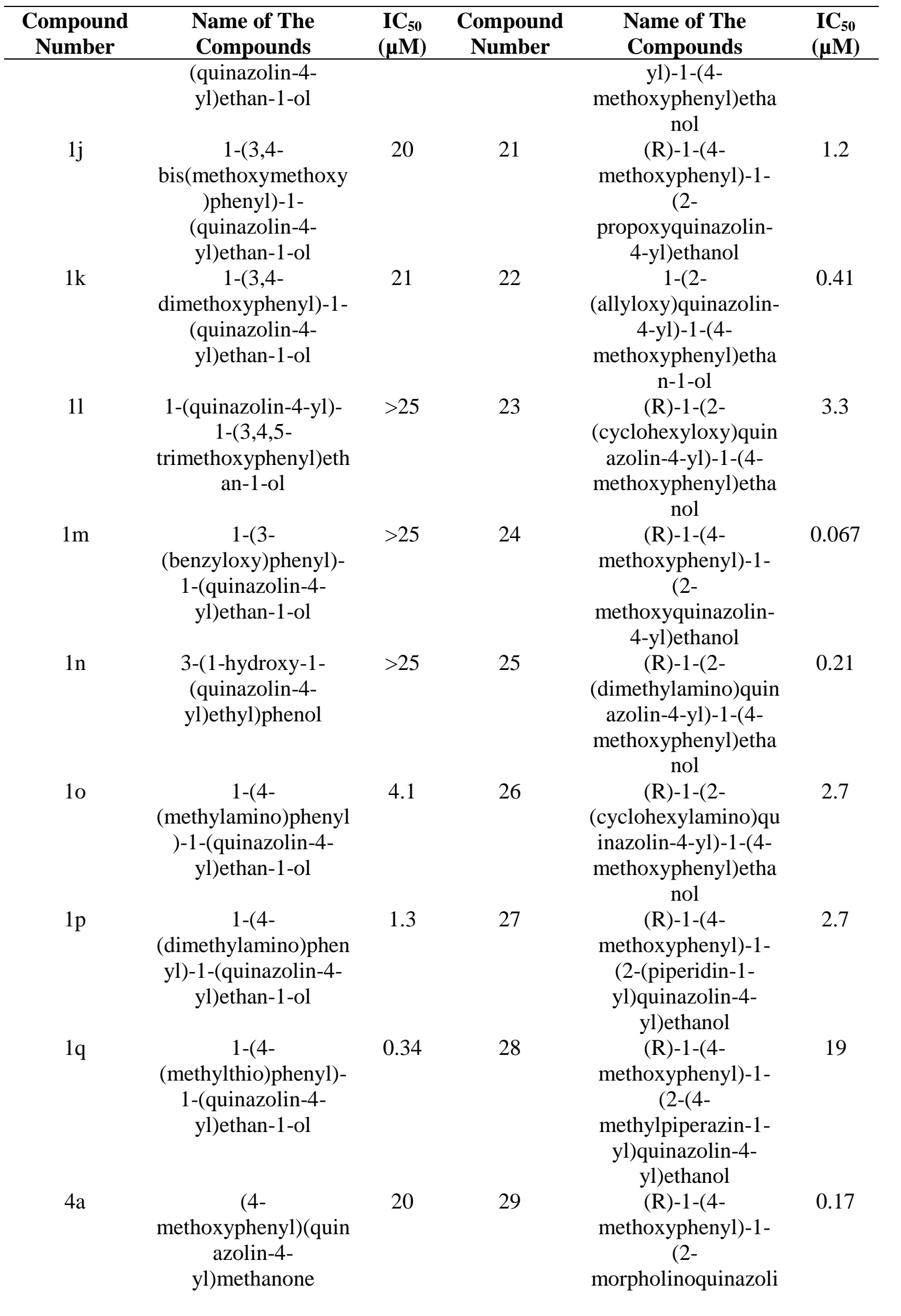




\begin{tabular}{|c|c|c|c|c|c|}
\hline $\begin{array}{c}\text { Compound } \\
\text { Number }\end{array}$ & $\begin{array}{l}\text { Name of The } \\
\text { Compounds }\end{array}$ & $\begin{array}{l}\mathrm{IC}_{50} \\
(\mu \mathrm{M})\end{array}$ & $\begin{array}{c}\text { Compound } \\
\text { Number }\end{array}$ & $\begin{array}{l}\text { Name of The } \\
\text { Compounds }\end{array}$ & $\begin{array}{l}\mathrm{IC}_{50} \\
(\mu \mathrm{M})\end{array}$ \\
\hline $4 \mathrm{f}$ & $\begin{array}{l}\text { (4- } \\
\text { ethoxyphenyl)(quina } \\
\text { zolin-4-yl)methanone }\end{array}$ & $>25$ & 30 & $\begin{array}{c}\text { n-4-yl)ethanol } \\
\text { (R)-1-(2-(4-(4- } \\
\text { fluorophenyl)piperaz } \\
\text { in-1-yl)quinazolin-4- } \\
\text { yl)-1-(4- } \\
\text { methoxyphenyl)etha } \\
\text { nol }\end{array}$ & 1.9 \\
\hline 5 & $\begin{array}{c}1-(4- \\
\text { methoxyphenyl)-1- } \\
\text { (quinazolin-4- } \\
\text { yl)propan-1-ol }\end{array}$ & 1.8 & 31 & $\begin{array}{l}\text { (1R)-1-(4- } \\
\text { methoxyphenyl)-1- } \\
\text { (2-(thiophen-3- } \\
\text { yl)quinazolin-4- } \\
\text { yl)ethanol }\end{array}$ & 0.035 \\
\hline 6 & $\begin{array}{l}(4- \\
\text { methoxyphenyl)(phe } \\
\text { nyl)(quinazolin-4- } \\
\text { yl)methanol }\end{array}$ & 9.8 & 32 & $\begin{array}{l}\text { (R)-1-(2-(4- } \\
\text { chlorophenyl)quinaz } \\
\text { olin-4-yl)-1-(4- } \\
\text { methoxyphenyl)etha } \\
\text { nol }\end{array}$ & 0.40 \\
\hline 7 & $\begin{array}{l}\text { 2,2,2-trifluoro-1-(4- } \\
\text { methoxyphenyl)-1- } \\
\text { (quinazolin-4- } \\
\text { yl)ethan-1-ol }\end{array}$ & 1.1 & 33 & $\begin{array}{c}\text { 1-(2-(2- } \\
\text { chlorophenyl)quinaz } \\
\text { olin-4-yl)-1-(4- } \\
\text { methoxyphenyl)etha } \\
\text { n-1-ol }\end{array}$ & 0.78 \\
\hline 8 & $\begin{array}{l}(4- \\
\text { methoxyphenyl)(quin } \\
\text { azolin-4-yl)methanol }\end{array}$ & $>25$ & 34 & $\begin{array}{c}\text { 1-(2-(3- } \\
\text { chlorophenyl)quinaz } \\
\text { olin-4-yl)-1-(4- } \\
\text { methoxyphenyl)etha } \\
\text { n-1-ol }\end{array}$ & 2.1 \\
\hline
\end{tabular}

\section{Methods}

The 2D structures of 44 compounds of 1-phenyl-1-(quinazolin-4-yl) ethanol were prepared using ChemDraw 2016. The 1-phenyl-1-(quinazoline-4-yl) ethanol compounds were screened using pkCSM to find out whether the compounds conform the Lipinski's rule of Five. The unconform compounds maximum 2 endpoints of Lipinski's rule of Five were eliminated. The toxicity of screened 1-phenyl-1-(quinazolin-4-yl) ethanol compound were predicted using Toxtree, pkCSM and PreADMET. The endpoints selected in Toxtree were Cramer rule, Kroes TTC decision tree, carcinogenicity (genotox and non genotox) mutagenicity rule base by ISS, and in vitro mutagenicity (Ames test) alerts by ISS. Open Babel GUI is used in pkCSM to create a compound SMILE format. The selected endpoint in pkCSM were Ames Toxicity, Maximum Tolerated Dose, Rat Acute Oral Toxicity $\left(\mathrm{LD}_{50}\right)$ and hepatotoxicity. The selected endpoints in PreADMET were Ames Test and Rodent Carcinogenicity (Mice and Rat).

\section{Data Analysis}

The toxicity prediction results of 1-phenyl-1-(quinazolin-4-yl) ethanol compounds were quantitative and qualitative data. Qualitative data were expressed in positive and negative statements. Then made in the form of scoring, where the positive toxic score was 1 and negative toxic scored was 2 . The data analysis used the scoring model by summing all endpoints of Toxtree, pkCSM and PreADMET to obtain five compounds with the lowest toxicity effect (largest score). Then five of 1-phenyl-1-(quinazolin-4-yl) ethanol compounds which have high activity based on in 
vitro test of Kuroiwa et al. (2015) against cell line A549 (lung) were selected. The best compound is obtained through the selected scoring model by comparing any compounds having a low toxic effect and followed by the most amount of toxic negative endpoints. The next step for getting the three compounds that have the highest activity with the lowest toxicity was comparing the highest scores and the smallest $\mathrm{IC}_{50}$ values among the five compounds.

\section{RESULTS AND DISCUSSION}

Lipinski's rule of five calculations

The Lipinski's rule of five calculations were performed to determine the degree of absorption or permeability of compounds against lipid bilayers in the human body. The Lipinski rule is a parameter that demonstrates the oral bioavailability of a compound. Good bioavailability will satisfy the Lipinski rule where the maximum molecular weight of the compound is 500 , the $\log \mathrm{P}$ is not greater than 5, the hydrogen bond donor is less than 5, and hydrogen bond acceptor is less than 10 (Lipinski et al., 2012). The results of Lipinski's rule of Five calculations using pkCSM are presented in Table II.

Table II. Results of Lipinski's Rule of Five calculation

\begin{tabular}{|c|c|c|c|c|}
\hline $\begin{array}{l}\text { Compound } \\
\text { Number }\end{array}$ & $\begin{array}{l}\text { Molecular } \\
\text { Weight }\end{array}$ & $\log P$ & $\begin{array}{c}\text { Hydrogen } \\
\text { Bonds Acceptor }\end{array}$ & $\begin{array}{c}\text { Hydrogen } \\
\text { Bonds } \\
\text { Donor } \\
\end{array}$ \\
\hline $1 \mathrm{a}$ & 280.327 & 2.8942 & 4 & 1 \\
\hline $1 b$ & 268.291 & 3.0247 & 3 & 1 \\
\hline $1 c$ & 284.746 & 3.5390 & 3 & 1 \\
\hline $1 d$ & 318.298 & 3.9044 & 3 & 1 \\
\hline $1 \mathrm{f}$ & 294.354 & 3.2843 & 4 & 1 \\
\hline $1 \mathrm{~g}$ & 322.408 & 4.0629 & 4 & 1 \\
\hline $1 \mathrm{~h}$ & 334.297 & 3.7842 & 4 & 1 \\
\hline $1 \mathrm{i}$ & 280.327 & 2.8942 & 4 & 1 \\
\hline $1 \mathrm{j}$ & 370.405 & 2.8510 & 7 & 1 \\
\hline $1 \mathrm{k}$ & 310.353 & 2.9028 & 5 & 1 \\
\hline 11 & 342.351 & 1.8413 & 7 & 2 \\
\hline $1 \mathrm{~m}$ & 356.425 & 4.4646 & 4 & 1 \\
\hline $1 \mathrm{n}$ & 266.300 & 2.5912 & 4 & 2 \\
\hline 10 & 295.386 & 3.5634 & 4 & 2 \\
\hline $1 \mathrm{p}$ & 293.370 & 2.9516 & 4 & 1 \\
\hline $1 \mathrm{q}$ & 296.395 & 3.6075 & 4 & 1 \\
\hline $4 a$ & 264.284 & 2.8694 & 4 & 0 \\
\hline $4 \mathrm{f}$ & 278.311 & 3.2595 & 4 & 0 \\
\hline 5 & 294.354 & 3.2843 & 4 & 1 \\
\hline 6 & 294.354 & 3.2843 & 4 & 1 \\
\hline 7 & 334.297 & 3.4366 & 4 & 1 \\
\hline 8 & 266.300 & 2.7201 & 4 & 1 \\
\hline 9 & 310.353 & 2.9028 & 5 & 1 \\
\hline
\end{tabular}

Pharmaciana Vol. 8, No. 2, Nov 2018, Page. 205 - 216 


\begin{tabular}{|c|c|c|c|c|}
\hline $\begin{array}{l}\text { Compound } \\
\text { Number }\end{array}$ & $\begin{array}{l}\text { Molecular } \\
\text { Weight }\end{array}$ & $\log P$ & $\begin{array}{c}\text { Hydrogen } \\
\text { Bonds Acceptor }\end{array}$ & $\begin{array}{c}\text { Hydrogen } \\
\text { Bonds } \\
\text { Donor } \\
\end{array}$ \\
\hline 14 & 294.354 & 3.2026 & 4 & 1 \\
\hline 15 & 362.473 & 4.9419 & 4 & 1 \\
\hline 16 & 397.689 & 4.7209 & 4 & 1 \\
\hline 17 & 314.772 & 3.5476 & 4 & 1 \\
\hline 18 & 296.326 & 2.1875 & 4 & 2 \\
\hline 19 & 310.353 & 2.9028 & 5 & 1 \\
\hline 20 & 324.380 & 3.2929 & 5 & 1 \\
\hline 21 & 338.407 & 3.6830 & 5 & 1 \\
\hline 22 & 352.434 & 4.0951 & 5 & 1 \\
\hline 23 & 378.472 & 4.6057 & 5 & 1 \\
\hline 24 & 326.421 & 3.6161 & 5 & 1 \\
\hline 25 & 323.396 & 2.9602 & 5 & 1 \\
\hline 26 & 377.488 & 4.6388 & 5 & 2 \\
\hline 27 & 363.461 & 3.8845 & 5 & 1 \\
\hline 28 & 378.476 & 2.6460 & 6 & 1 \\
\hline 29 & 365.433 & 2.7308 & 6 & 1 \\
\hline 30 & 458.537 & 4.3599 & 6 & 1 \\
\hline 31 & 362.454 & 4.6227 & 5 & 1 \\
\hline 32 & 390.870 & 5.2146 & 4 & 1 \\
\hline 33 & 390.870 & 5.2146 & 4 & 1 \\
\hline 34 & 390.870 & 5.2146 & 4 & 1 \\
\hline
\end{tabular}

Based on results of Lipinski's Rule of Five calculations, all of 1-phenyl-1-(quinazolin-4-yl) ethanol compounds conform Lipinski's rule. All compounds were predicted having good absorptivity for an oral medication (Wulandari and Kristin, 2010). Based on research conducted by Veber et al. (2002) concluded that a compound with lower molecular weight, log P, hydrogen bond donor, and hydrogen bond acceptor has the higher bioavailability.

\section{Toxicity predictions} III.

The results of toxicity prediction by Toxtree, pkCSM and PreADMET are presented in Table

Table III. Results of toxicity prediction by Toxtree, pkCSM and PreADMET

\begin{tabular}{cccccccccccccc}
\hline $\begin{array}{c}\text { Compound } \\
\text { Number }\end{array}$ & $\mathbf{A}$ & $\mathbf{B}$ & $\mathbf{C}$ & $\mathbf{D}$ & $\mathbf{E}$ & $\mathbf{F}$ & $\mathbf{G}$ & $\mathbf{H}$ & $\mathbf{I}$ & $\mathbf{J}$ & $\mathbf{K}$ & $\mathbf{L}$ & Scoring \\
\hline 1a & 1 & 1 & 2 & 2 & 2 & 2 & 1.273 & 2.079 & 2 & 1 & 2 & 2 & 20.352 \\
1b & 1 & 1 & 2 & 1 & 2 & 1 & 1.330 & 2.016 & 2 & 1 & 1 & 2 & 17.346 \\
1c & 1 & 1 & 2 & 2 & 2 & 1 & 1.225 & 1.973 & 2 & 2 & 1 & 2 & 19.198 \\
1d & 1 & 1 & 2 & 2 & 2 & 2 & 1.072 & 2.223 & 1 & 2 & 1 & 2 & 19.295 \\
1f & 1 & 1 & 2 & 2 & 2 & 1 & 2.323 & 1.870 & 2 & 1 & 2 & 2 & 20.193 \\
1g & 1 & 1 & 2 & 2 & 2 & 2 & -1.119 & 2.007 & 1 & 2 & 2 & 2 & 17.888
\end{tabular}




\begin{tabular}{|c|c|c|c|c|c|c|c|c|c|c|c|c|c|}
\hline $\begin{array}{c}\text { Compound } \\
\text { Number }\end{array}$ & A & B & C & D & $\mathbf{E}$ & $\mathbf{F}$ & G & H & I & $\mathbf{J}$ & $\mathbf{K}$ & $\mathbf{L}$ & Scoring \\
\hline $1 \mathrm{~h}$ & 1 & 1 & 2 & 2 & 2 & 2 & -1.127 & 2.295 & 2 & 1 & 2 & 2 & 18.168 \\
\hline $1 \mathrm{i}$ & 1 & 1 & 2 & 2 & 2 & 2 & 1.849 & 1.834 & 2 & 1 & 1 & 2 & 19.683 \\
\hline $1 \mathrm{j}$ & 1 & 1 & 2 & 2 & 2 & 2 & 3.034 & 2.204 & 2 & 2 & 2 & 2 & 23.238 \\
\hline $1 \mathrm{k}$ & 1 & 1 & 2 & 2 & 2 & 2 & 1.766 & 2.269 & 1 & 2 & 2 & 2 & 21.035 \\
\hline 11 & 1 & 1 & 2 & 2 & 2 & 2 & 2.265 & 1.906 & 1 & 2 & 2 & 2 & 21.171 \\
\hline $1 \mathrm{~m}$ & 1 & 1 & 2 & 2 & 2 & 1 & 2.265 & 2.466 & 1 & 1 & 1 & 2 & 18.731 \\
\hline $1 \mathrm{n}$ & 1 & 1 & 2 & 2 & 2 & 2 & 1.442 & 2.147 & 2 & 1 & 1 & 2 & 19.589 \\
\hline 10 & 1 & 2 & 1 & 2 & 1 & 2 & 1.528 & 2.367 & 2 & 2 & 1 & 2 & 19.895 \\
\hline $1 p$ & 1 & 2 & 1 & 2 & 1 & 2 & 1.442 & 2.147 & 2 & 2 & 1 & 2 & 19.589 \\
\hline $1 q$ & 1 & 1 & 2 & 2 & 2 & 2 & 2.094 & 1.804 & 2 & 1 & 1 & 2 & 19.898 \\
\hline $4 a$ & 1 & 1 & 2 & 2 & 2 & 2 & 1.879 & 2.294 & 2 & 1 & 2 & 2 & 21.173 \\
\hline $4 \mathrm{f}$ & 1 & 1 & 2 & 2 & 2 & 1 & 4.188 & 2.245 & 2 & 1 & 2 & 2 & 22.433 \\
\hline 5 & 1 & 1 & 2 & 2 & 2 & 2 & 1.227 & 2.116 & 2 & 2 & 2 & 2 & 21.343 \\
\hline 6 & 1 & 1 & 2 & 2 & 2 & 1 & 2.275 & 2.996 & 1 & 2 & 2 & 2 & 21.271 \\
\hline 7 & 1 & 1 & 2 & 2 & 2 & 2 & 1.259 & 2.275 & 2 & 1 & 2 & 2 & 20.534 \\
\hline 8 & 1 & 1 & 2 & 2 & 2 & 2 & 1.164 & 1.980 & 2 & 1 & 2 & 2 & 20.144 \\
\hline 9 & 1 & 1 & 2 & 2 & 2 & 2 & 2.565 & 2.525 & 1 & 2 & 2 & 2 & 22.090 \\
\hline 14 & 1 & 1 & 2 & 2 & 2 & 2 & 1.361 & 2.525 & 2 & 2 & 2 & 2 & 21.886 \\
\hline 15 & 1 & 1 & 2 & 1 & 2 & 2 & -1.349 & 2.179 & 1 & 2 & 2 & 2 & 16.830 \\
\hline 16 & 1 & 1 & 2 & 2 & 2 & 2 & 1.432 & 2.641 & 2 & 2 & 2 & 2 & 22.073 \\
\hline 17 & 1 & 1 & 2 & 2 & 2 & 2 & 1.208 & 2.270 & 1 & 2 & 2 & 2 & 20.478 \\
\hline 18 & 1 & 1 & 2 & 2 & 2 & 2 & 2.005 & 2.139 & 2 & 1 & 1 & 2 & 20.144 \\
\hline 19 & 1 & 1 & 2 & 2 & 2 & 2 & 1.503 & 2.189 & 2 & 2 & 2 & 2 & 21.692 \\
\hline 20 & 1 & 1 & 2 & 2 & 2 & 2 & 1.496 & 2.237 & 1 & 2 & 2 & 2 & 20.733 \\
\hline 21 & 1 & 1 & 2 & 2 & 2 & 2 & 3.169 & 2.091 & 1 & 2 & 2 & 2 & 22.260 \\
\hline 22 & 1 & 1 & 2 & 2 & 2 & 2 & 2.606 & 2.231 & 2 & 1 & 2 & 2 & 21.837 \\
\hline 23 & 1 & 1 & 2 & 2 & 2 & 2 & 1.334 & 2.472 & 1 & 2 & 2 & 2 & 20.806 \\
\hline 24 & 1 & 1 & 1 & 2 & 2 & 2 & 1.337 & 2.170 & 2 & 2 & 2 & 2 & 20.507 \\
\hline 25 & 1 & 2 & 1 & 2 & 1 & 2 & 1.170 & 2.141 & 1 & 2 & 2 & 2 & 19.311 \\
\hline 26 & 1 & 1 & 2 & 2 & 2 & 2 & -1.462 & 2.539 & 1 & 2 & 2 & 2 & 18.077 \\
\hline 27 & 1 & 1 & 2 & 2 & 2 & 2 & -1.459 & 2.326 & 1 & 2 & 2 & 2 & 17.867 \\
\hline 28 & 1 & 1 & 2 & 2 & 2 & 2 & 1.288 & 2.617 & 1 & 2 & 1 & 2 & 19.905 \\
\hline 29 & 1 & 1 & 2 & 2 & 2 & 2 & -1.049 & 2.245 & 1 & 2 & 2 & 2 & 18.196 \\
\hline 30 & 1 & 1 & 2 & 2 & 2 & 2 & 1.119 & 2.541 & 1 & 2 & 2 & 2 & 20.660 \\
\hline 31 & 1 & 1 & 2 & 1 & 2 & 1 & 2.512 & 2.289 & 1 & 2 & 2 & 2 & 19.801 \\
\hline 32 & 1 & 1 & 2 & 1 & 2 & 1 & 2.576 & 2.397 & 1 & 2 & 2 & 2 & 19.973 \\
\hline 33 & 1 & 1 & 2 & 1 & 2 & 1 & 2.600 & 2.424 & 1 & 2 & 2 & 2 & 20.024 \\
\hline 34 & 1 & 1 & 2 & 1 & 2 & 1 & 2.431 & 2.431 & 1 & 2 & 2 & 2 & 16.862 \\
\hline
\end{tabular}


Information:

$1=$ Positive toxic

$2=$ Negative toxic

$\mathrm{A}=$ Cramer rule Toxtree

$\mathrm{B}=$ Kroes TTC Toxtree

$\mathrm{C}=$ Carcinogenicity genotox Toxtree

$\mathrm{D}=$ Carcinogenicity non genotox Toxtree

$\mathrm{E}=$ In vitro mutagenicity (Ames test) Toxtree

$\mathrm{F}=$ Mutagenicity (Ames test) pkCSM
$\mathrm{G}=$ Maximum daily dose pkCSM ( $\mathrm{mg} / \mathrm{kg} /$ day)

$\mathrm{H}=$ Rat Acute Oral Toxicity $\left(\mathrm{LD}_{50}\right) \mathrm{pkCSM}$ $(\mathrm{mol} / \mathrm{kg})$

$\mathrm{I}=$ Hepatotoxicity pkCSM

$\mathrm{J}=$ Mutagenicity (Ames Test) PreADMET

$\mathrm{K}=$ Carcinogenicity to rat PreADMET

$\mathrm{L}=$ Carcinogenicity to mice PreADMET

Table IV. Classification of the compounds based on their toxicity

\begin{tabular}{|c|c|}
\hline Toxicity & Compound \\
\hline $\begin{array}{l}\text { High toxicity risk (Cramer } \\
\text { rules Toxtree) }\end{array}$ & $\begin{array}{l}\text { 1a, 1b, 1c, 1d, 1f, 1g, 1h, 1i, 1j, 1k, 11, 1m, 1n, 1o, 1p, 1q, 4a, 4f, 5, 6, } \\
7,8,9,14,15,16,17,18,19,20,21,22,23,24,25,26,27,28,29, \\
30,31,32,33 \text { and } 34\end{array}$ \\
\hline $\begin{array}{l}\text { High toxicity risk (Kroes } \\
\text { TTC Toxtree) }\end{array}$ & $\begin{array}{l}\text { la, } 1 \mathrm{~b}, 1 \mathrm{c}, 1 \mathrm{~d}, 1 \mathrm{f}, 1 \mathrm{~g}, 1 \mathrm{~h}, 1 \mathrm{i}, 1 \mathrm{j}, 1 \mathrm{k}, 1 \mathrm{l}, 1 \mathrm{~m}, 1 \mathrm{n}, 1 \mathrm{q}, 4 \mathrm{a}, 4 \mathrm{f}, 5,6,7,8,9 \text {, } \\
14,15,16,17,18,19,20,21,22,23,24,26,27,28,29,30,31,32,33 \\
\text { and } 34\end{array}$ \\
\hline $\begin{array}{l}\text { Carcinogen genotox } \\
\text { Toxtree }\end{array}$ & $1 \mathrm{o}, 1 \mathrm{p}, 24$ and 25 \\
\hline $\begin{array}{l}\text { Carcinogen non genotox } \\
\text { Toxtree }\end{array}$ & $1 b, 31,32,33$ and 34 \\
\hline $\begin{array}{l}\text { In vitro mutagen (Ames } \\
\text { test) Toxtree }\end{array}$ & $1 \mathrm{o}, 1 \mathrm{p}, 25$ and 26 \\
\hline $\begin{array}{l}\text { Mutagen (Ames test) } \\
\text { pkCSM }\end{array}$ & $1 \mathrm{~b}, 1 \mathrm{c}, 1 \mathrm{f}, 1 \mathrm{~m}, 4 \mathrm{f}, 6,31,32,33$ and 34 \\
\hline $\begin{array}{l}\text { The lowest maximum } \\
\text { daily dose pkCSM }\end{array}$ & 26 \\
\hline $\begin{array}{l}\text { The lowest rat acute oral } \\
\text { toxicity (LD50) pkCSM }\end{array}$ & $1 \mathrm{q}$ \\
\hline Hepatotoxic pkCSM & $\begin{array}{l}1 \mathrm{~d}, 1 \mathrm{~g}, 1 \mathrm{k}, 11,1 \mathrm{~m}, 6,9,15,17,20,21,23,25,26,27,28,29,30,31 \text {, } \\
32,33 \text { and } 34\end{array}$ \\
\hline $\begin{array}{l}\text { Mutagen (Ames Test) } \\
\text { PreADMET }\end{array}$ & $1 \mathrm{a}, 1 \mathrm{~b}, 1 \mathrm{f}, 1 \mathrm{~h}, 1 \mathrm{i}, 1 \mathrm{~m}, 1 \mathrm{n}, 1 \mathrm{q}, 4 \mathrm{a}, 4 \mathrm{f}, 7,8,18$ and 22 \\
\hline $\begin{array}{l}\text { Carcinogen to rat } \\
\text { PreADMET }\end{array}$ & $1 \mathrm{~b}, 1 \mathrm{c}, 1 \mathrm{~d}, 1 \mathrm{i}, 1 \mathrm{~m}, 1 \mathrm{n}, 1 \mathrm{o}, 1 \mathrm{p}, 1 \mathrm{q}, 18$ and 28 \\
\hline
\end{tabular}

According to Table III and Table IV, in the Cramer rule Toxtree, all of 1-phenyl-1(quinazolin-4-yl) ethanol compound derivatives are class 3 (score 1) which have a high toxicity risk. It means the high concentrations of the 1-phenyl-1-(quinazolin-4-yl) ethanol compounds are not guaranteed for the safety in their use. Based on the Kroes TTC endpoint, 41 compounds are positively at high risk bacause their exposure limits more than $0.15 \mu \mathrm{g} /$ day (score 1). While compounds $1 \mathrm{o}, 1 \mathrm{p}$, and 25 have no a significant risks (score 2). The risk can be reduced if given at or below $0.15 \mu \mathrm{g} /$ day with a threshold value of $86-97 \%$. Based on the predictions of carcinogenicity (genotoxic and non genotoxic), compounds $1 \mathrm{o}, 1 \mathrm{p}, 24$, and 25 are genotoxic carcinogenic (score 1) whereas compounds $1 \mathrm{~b}, 31,32,33$, and 34 are non-genotoxic carcinogenic (score 1). Genotoxic carcinogens cause irreversible genetic damage or mutations by binding to DNA. Non-genotoxic carcinogens or epigenetics not bind covalently to DNA do not cause DNA damage directly and generally negative for mutagenicity tests. Based on in vitro mutagenicity (Ames test) predictions, compounds 1o, 1p, 25, and 26 have risk as mutagen (score 1), while 40 other compounds have no risk as mutagen (score 2). 
Compound 6 has the highest value of $\mathrm{LD}_{50}$ endpoint of $2.996 \mathrm{~mol} / \mathrm{kg}$. Compound $4 \mathrm{f}$ has the highest value at the maximum daily dose endpoint of $4.188 \mathrm{mg} / \mathrm{kg} / \mathrm{day}$. The higher maximum daily dose and $\mathrm{LD}_{50}$ value in the acute toxicity test of the compound, the compound will not have toxic effect on the mice. Based on the mutagenicity endpoint of pKCSM, the compounds 1b, 1c, 1f, 1m, 4f, 6, 31, 32, 33 and 34 are mutagenic (score 1) whereas the other compounds are non-mutagenic (score 2). The last parameter of pkCSM is hepatotoxic. There are 22 hepatotoxic compounds (score 1) and 22 non-hepatotoxic compounds (score 2).

At the Ames test endpoint of PreADMET, there are 14 mutagenic compounds (score 1) and 30 other compounds are non-mutagenic compounds (score 2). The positive test results on Ames test indicate that the compound is mutagenic and has the possibility as carcinogenic. In the prediction of carcinogenicity in rat produced 11 carcinogenic positive compounds (score 1) and 33 other compounds are negative carcinogenic (score 2 ). While in the prediction of carcinogenicity in mice, all of compounds are not carcinogenicity (score 2).

In the study of Kuroiwa et al. (2015) obtained compounds 14, 16, 17, 19 and 31 which have the best activity with $\mathrm{IC}_{50} 0.053 \mu \mathrm{M}, 0.038 \mu \mathrm{M}, 0.027 \mu \mathrm{M}, 0.058 \mu \mathrm{M}$ and $0.035 \mu \mathrm{M}$ on cell line A549 (lung). The results of the toxicity prediction showed the five compounds having the lowest toxicity (largest scores), i.e. compounds 1j, 5, 14, 16 and 19. Compound 31 was not selected because of hepatotoxic, non-genotoxic and mutagenic carcinogens. While the compound 17 was not selected because of hepatotoxic. Compounds 1j, 5, 14, 16 and 19 are negative genotoxic carcinogens and non-genotoxic carcinogens, in vitro mutagenicity (Ames test), hepatotoxicity, and carcinogenicity in mice and rat. The highest maximum daily dose value among the five compounds is compound $1 \mathrm{j}$, that is $3.034 \mathrm{mg} / \mathrm{kg} /$ day while the highest $\mathrm{LD}_{50}$ value is compound 16 , that is 2.642 $\mathrm{mol} / \mathrm{kg}$.

Based on in vitro test results Kuroiwa et al. (2015) compounds 1j, 5, 14, 16 and 19 have $\mathrm{IC}_{50}$ values of $20 \mu \mathrm{M}, 1.8 \mu \mathrm{M}, 0.053 \mu \mathrm{M}, 0.038 \mu \mathrm{M}$ and $0.058 \mu \mathrm{M}$. The compound with smaller $\mathrm{IC}_{50}$ is a compound that has higher activity as anticancer. Compounds $1 \mathrm{j}$ and 5 are not selected because they have a lower activity value compared to the other three compounds. Compounds that have

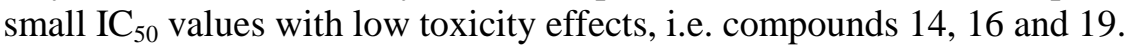

\section{CONCLUSION}

In the toxicity prediction of 1-phenyl-1-(quinazolin-4-yl) ethanol compounds using Toxtree, pkCSM and preADMET, three anticancer compounds have the highest activity in A549 cell (lung) and lowest toxicity i.e. compounds 14,16 and 19 . Compound 14 has $\mathrm{IC}_{50}$ of $0.053 \mu \mathrm{M}$ and toxicity score of 21.886. Compound 16 has $\mathrm{IC}_{50}$ of $0.038 \mu \mathrm{M}$ and toxicity score of 22.073. Meanwhile, compound 19 has $\mathrm{IC}_{50}$ of $0.058 \mu \mathrm{M}$ and toxicity score of 21.692 .

\section{REFERENCES}

Ajani, O. O., Aderohunmu, D. V., Umeokoro, E. N. and Olomieja, A. O., 2016. Quinazoline pharmacophore in therapeutic medicine, Bangladesh Journal of Pharmacology, 11(3): 716733.

Benigni, R., Bossa, C., Jeliazkova, N., Netzeva, T. and Worth, A., 2008. The Benigni/Bossa Rulebase for Mutagenicity and Carcinogenicity-A Module of Toxtree. JRC Scientific and Technical Reports, Retrieved from http://ihcp.jrc.ec.europa.eu/our_labs/ computational_toxicology/doc/EUR_23241_EN.pdf.

Faraj, F.L., Zahedifard, M., Paydar, M., Looi, C.Y., Abdul Majid, N., Ali, H.M., Ahmad, N., Gwaram, N.S., Abdulla, M.A., Faraj, F.L., Zahedifard, M., Paydar, M., Looi, C.Y., Abdul Majid, N., Ali, H.M., Ahmad, N., Gwaram, N.S., Abdulla, M.A., 2014. Synthesis, characterization, and anticancer activity of new quinazoline derivatives against MCF-7 cells. The Scientific World Journal, 2014: 1-15.

Kuroiwa, K., Ishii, H., Matsuno, K., Asai, A. and Suzuki, Y., 2015. Synthesis and structure-activity 
relationship study of 1-phenyl-1-(quinazolin-4-yl)ethanols as anticancer agents. ACS Medicinal Chemistry Letters, 6(3): 287-291.

Lipinski, C. A., Lombardo, F., Dominy, B. W. and Feeney, P. J., 2012. Experimental and computational approaches to estimate solubility and permeability in drug discovery and development settings, Advanced Drug Delivery Reviews, 64(SUPPL.): 4-17.

Parameshwar, R., Harinadha Babu, V., Manichandrika, P., Narendra Sharath Chandra, J. N. and Swetha, K., 2016. Design, synthesis, in silico toxicity prediction, molecular docking, and evaluation of novel pyrazole derivatives as potential antiproliferative agents, EXCLI Journal, 15: 187-202.

Pires, D. E. V., Blundell, T. L. and Ascher, D. B., 2015. pkCSM: Predicting small-molecule pharmacokinetic and toxicity properties using graph-based signatures. Journal of Medicinal Chemistry, 58(9): 4066-4072.

Priyanto, 2015.Toksikologi, mekanisme, terapi antidotum, dan penilaian resikoi. Depok: Lembaga Studi dan Konsultasi Farmakologi (Leskonfi).

Raies, A. B. and Bajic, V. B., 2016. In Silico Toxicology: Computational methods for the prediction of chemical toxicity, Wiley Interdisciplinary Reviews: Computational Molecular Science, 6: 147-172.

Riju, A., Sithara, K., Nair, S. S. and Eapen, S. J., 2010. Prediction of toxicity and pharmacological potential of selected spice compounds. In Proceedings of the International Symposium on Biocomputing, 31:1-15.

Valerio, L. G., 2009. In silico toxicology for the pharmaceutical sciences. Toxicology and Applied Pharmacology, 241(3): 356-370.

Veber, D. F., Johnson, S. R., Cheng, H., Smith, B. R., Ward, K. W. and Kopple, K. D., 2002. Molecular properties that influence the oral bioavailability of drug candidates, Journal of Medicinal Chemistry, 45: 2615-2623.

Wulandari and Kristin, E., 2010. Analisis interaksi histone deacetylase (HDAC) kelas II Homo Sapiens dengan Suberoyllanilide Hydroxamic Acids (SAHA) dan Trichostatin A (TSA). Tesis, Depok: Fakultas Matematika dan Ilmu Pengetahuan Alam UI.

Zhang, L., Ai, H., Chen, W., Yin, Z., Hu, H., Zhu, J., Zhao, J., Zhao, Q., Liu, H., 2017. CarcinoPred-EL: Novel models for predicting the carcinogenicity of chemicals using molecular fingerprints and ensemble learning methods, Scientific Reports, 7(1):1-14. 
